\title{
Average fast neutron flux in three energy ranges in the Quinta assembly irradiated by two types of beams
}

\author{
Elzbieta Strugalska-Gola ${ }^{1, \star}$, Marcin Bielewicz ${ }^{1,2, \star \star}$, Stanislaw Kilim ${ }^{1}$, Marcin Szuta $^{1}$, and \\ Sergey Tyutyunnikov ${ }^{2}$ \\ ${ }^{1}$ National Centre for Nuclear Research, 05-400 Otwock-Świerk, Poland \\ ${ }^{2}$ Joint Institute for Nuclear Research, 141980 Dubna, Russia
}

\begin{abstract}
This work was performed within the international project "Energy plus Transmutation of Radioactive Wastes" (E\&T - RAW) for investigations of energy production and transmutation of radioactive waste of the nuclear power industry. ${ }^{89} \mathrm{Y}$ (Yttrium 89) samples were located in the Quinta assembly in order to measure an average high neutron flux density in three different energy ranges using deuteron and proton beams from Dubna accelerators. Our analysis showed that the neutron density flux for the neutron energy range $20.8-32.7 \mathrm{MeV}$ is higher than for the neutron energy range $11.5-20.8 \mathrm{MeV}$ both for protons with an energy of $0.66 \mathrm{GeV}$ and deuterons with an energy of $2 \mathrm{GeV}$, while for deuteron beams of 4 and $6 \mathrm{GeV}$ we did not observe this.
\end{abstract}

\section{Introduction - Motivation}

Up to now, we have been concentrated on measuring the neutron flux distribution in the deeply subcritical Quinta assembly versus the axis and radius of the assembly for the neutron energy above $10 \mathrm{MeV}$ applying proton and deuteron beams of an energy from $1 \mathrm{GeV}$ to $8 \mathrm{GeV}$ extracted from the NUCLOTRON accelerator. We have applied a proton beam of an energy of $0.66 \mathrm{GeV}$ recently extracted from the PHASOTRON accelerator. Nuclear data handling at the experiment session have turned our attention to the neutron flux density measured on five foil plates in terms of three different neutron energies: $11.5-20.8 \mathrm{MeV}, 20.8-32.7 \mathrm{MeV}$ and $32.7-100 \mathrm{MeV}$. The neutron density flux for the neutron energy range $20.8-32.7 \mathrm{MeV}$ being higher than for the range $11.5-20.8 \mathrm{MeV}$ has appeared an unexpected feature of the measurement. Repeating in the same way data handling for the other planes as in collecting them in one figure it can be noticed that the same effect is observed for planes 2 - 5 but except plane 1, which describes the average neutron flux density in the first section where process of spallation begins. This unexpected feature of the measurement is presented in figure 1.

Finding this unexpected feature of an average neutron flux in the experiment with the proton beam of an energy of $0.66 \mathrm{GeV}$ motivated us to make an overview of our experiments performed earlier for the deuteron beam of an energy of $1-8 \mathrm{GeV}$.

\footnotetext{
$\star$ e-mail: elzbieta.strugalska-gola@ncbj.gov.pl

$\star \star$ e-mail: marcin.bielewicz@ncbj.gov.pl
} 


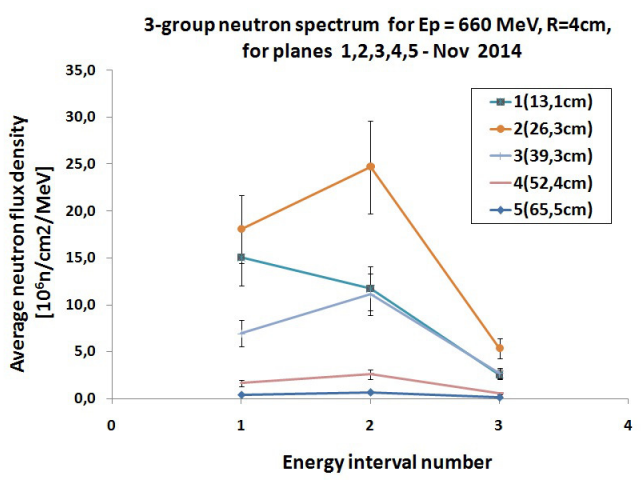

Figure 1. Average neutron flux density versus three different energy ranges on the $X$ axis: $1-(11.5-20.8 \mathrm{MeV})$, 2 - (20.8-32 MeV), 3 - (32-100 MeV) on five planes (legend on the right side of the figure); $\mathrm{R}=4 \mathrm{~cm}$, proton beam energy $0.66 \mathrm{GeV}$, Experiment: Nov 2014.

\section{Principles of the measurement}

To study the spectrum of high-energy neutrons, we have used threshold ${ }^{89} \mathrm{Y}$ detectors which have the following advantages: one stable isotope, several threshold reaction channels, several resulting isotopes with a half life time long enough - longer than 12 hours. The first threshold energy of the reaction (n, 2n) giving ${ }^{88} \mathrm{Y}$ has been for an energy of neutrons equal to $11.5 \mathrm{MeV}$. The next possible threshold energies 20.8, 32.7, 42.1 and $54.4 \mathrm{MeV}$ have been for the reactions $(\mathrm{n}, 3 \mathrm{n}){ }^{87} \mathrm{Y},(\mathrm{n}, 4 \mathrm{n}){ }^{86} \mathrm{Y}$, $(\mathrm{n}, 5 \mathrm{n}){ }^{85} \mathrm{Y}$ and $(\mathrm{n}, 6 \mathrm{n}){ }^{84} \mathrm{Y}$ respectively [1]. The neutron field has been determined with a certain number of ${ }^{89} \mathrm{Y}$ foils placed in specified positions (given by the radial and axial distance) inside the experimental facility (figure 2). After neutron irradiation, the gamma activity of ${ }^{89} \mathrm{Y}$ foils has been measured with an HPGe spectrometer. Taking into account necessary corrections [1] and spectra analysis by the DEIMOS program [2], we have determined isotope production per one gram of a sample and per one beam deuteron/proton at specified positions of the Quinta assembly.

Having isotope production determined inside of the experimental facility for the three isotopes ${ }^{88} \mathrm{Y},{ }^{87} \mathrm{Y}$ and ${ }^{86} \mathrm{Y}$, we have been able to evaluate three average high-energy neutron fluxes in each ${ }^{89} \mathrm{Y}$ foil location for certain energy ranges [3]. The energy ranges have been roughly determined by the microscopic cross section in function of the energy for the (n, xn) reactions of the three isotopes ${ }^{88} \mathrm{Y},{ }^{87} \mathrm{Y}$ and ${ }^{86} \mathrm{Y}$. The following three threshold energies 11.5, 20.8 and $32.7 \mathrm{MeV}$ for the reactions $(\mathrm{n}, 2 \mathrm{n}){ }^{89} \mathrm{Y}{ }^{88} \mathrm{Y},{ }^{89} \mathrm{Y}(\mathrm{n}, 3 \mathrm{n}){ }^{87} \mathrm{Y}$ and ${ }^{89} \mathrm{Y}(\mathrm{n}, 4 \mathrm{n}){ }^{86} \mathrm{Y}$ have appointed the first two energy ranges $(11.5$ - $20.8 \mathrm{MeV})$ and $(20.8-32.7 \mathrm{MeV})$ of the neutron fluxes $\bar{\Phi}_{1}$ and $\bar{\Phi}_{2}$. The third energy range $\bar{\Phi}_{3}$ has begun at $32.7 \mathrm{MeV}$ and ends at $100 \mathrm{MeV}$, when the microscopic cross section has been low comparatively to the maximum cross section.

The Quinta assembly, located at the Joint Institute for Nuclear Research (JINR), Dubna, Russia is an assembly of a massive uranium target and lead shielding (figure 2). The Quinta assembly consists of a total of $512 \mathrm{~kg}$ of natural uranium. It is composed of five sections, each being $114 \mathrm{~mm}$ long and separated by a $17 \mathrm{~mm}$ air gap, which allows the placement of samples mounted onto special plates. The uranium exists as many cylindrical rods, where each rod is $36 \mathrm{~mm}$ in diameter, $104 \mathrm{~mm}$ in length and $1.72 \mathrm{~kg}$ in mass. The first section contains only 54 rods after removal of the central 7 rods for creating a beam window. This beam window is $80 \mathrm{~mm}$ in diameter and serves to reduce the loss of backward emitted/scattered neutrons. The front and back of each section are bounded then by additional aluminum plates $350 \times 350 \times 5 \mathrm{~mm}$. The five sections are mounted onto a single slab of 

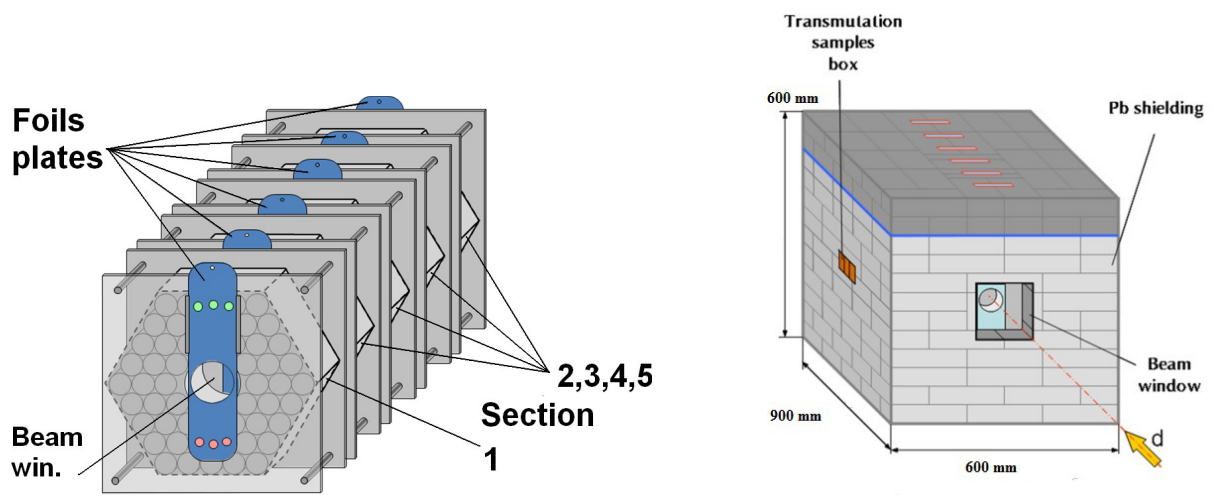

Figure 2. Scheme of the Quinta assembly. On the left, there is a view of the uranium target with supporting structures and plastics used for sample placing (foils plates), on the right, there is a view of the lead shielding enfolding the target.

aluminum with a thickness of $25 \mathrm{~mm}$ and surrounded by $100 \mathrm{~mm}$ thick lead bricks (shielding) on all six sides of a total weight of $1780 \mathrm{~kg}$. The sample plates are labeled 1-6, starting from the direction of the incident beam [4]-[5].

\section{Overview of experiments for the deuteron beam with an energy of 2 - 6 GeV}

Repeating data handling in the same way as in figure 1 for the deuteron beam with an energy of 2 $\mathrm{GeV}$ shows (figure 3) that the same effect is observed for planes 2, 4 and 5 and is not clear for planes 1 and 3 which describes the average neutron flux density in the first and third sections.

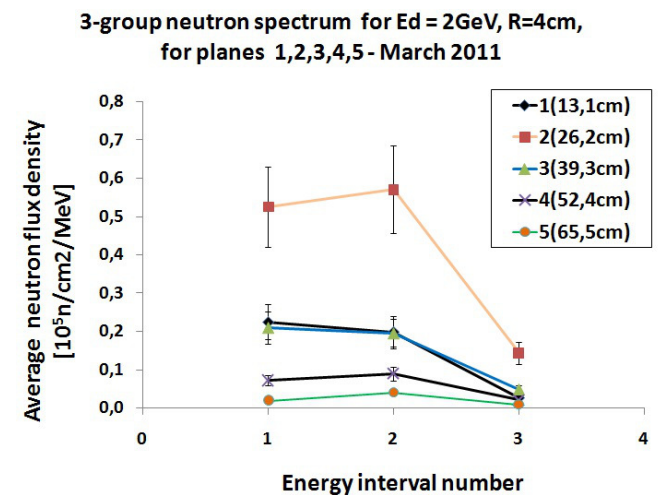

Figure 3. Average neutron flux density versus three different energy ranges on the $\mathrm{X}$ axis: $1-(11.5-20.8 \mathrm{MeV})$, 2 - (20.8-32 MeV), 3 - (32-100 MeV) on five planes (legend on the right side of the figure); $\mathrm{R}=4 \mathrm{~cm}$, deuteron beam energy $2 \mathrm{GeV}$, experiment III. 2011.

However the average neutron flux density versus three different energy ranges: 1 - (11.5$20.8 \mathrm{MeV}), 2$ - (20.8-32 MeV), 3 - (32-100 MeV) for $\mathrm{R}=4 \mathrm{~cm}$ and five planes for the deuteron beam 


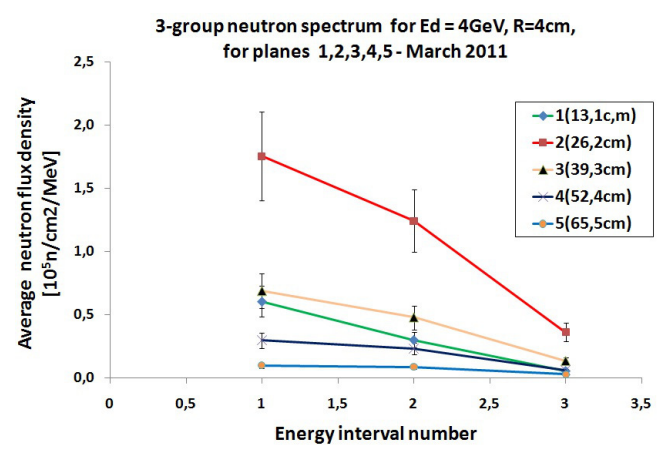

Figure 4. Average neutron flux density versus three different energy ranges on the $\mathrm{X}$ axis: 1- (11.5-20.8 MeV), 2- $(20.8-32 \mathrm{MeV}), 3-(32-100 \mathrm{MeV})$ on five planes (legend on the right side of the figure); $\mathrm{R}=4 \mathrm{~cm}$, deuteron beam energy $4 \mathrm{GeV}$, experiment III. 2011.

with an energy of $4 \mathrm{GeV}$ behaves as expected (figure 4). In this case, the explanation like for the experimental data in figure 1,3 is not sufficient.

Also the average neutron flux density versus three different energy ranges: 1 - (11.5-20.8 MeV), 2 - (20.8-32 MeV), 3 - (32-100 MeV) for $\mathrm{R}=4 \mathrm{~cm}$ and five planes for the deuteron beam with an energy of $6 \mathrm{GeV}$ behaves as expected (figure 5).

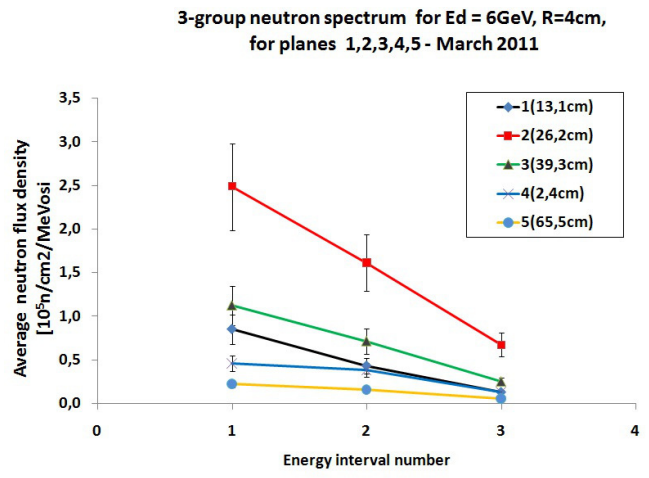

Figure 5. Average neutron flux density versus three different energy ranges on the $\mathrm{X}$ axis: 1- (11.5-20.8 MeV), 2- (20.8-32 MeV), 3- (32-100 MeV) on five planes (legend on the right side of the figure); $\mathrm{R}=4 \mathrm{~cm}$, deuteron beam energy $6 \mathrm{GeV}$, experiment III. 2011.

\section{Discussion}

Such an unexpected behavior of the average neutron flux density versus three different energy ranges can be explained to some extent by the dependence of the micro cross section in the considered neutron energy range shown in figure 1 and figure 3 . The threshold neutron energies of yttrium (n,xn) reactions determine the considered energy ranges.

In the first energy range $11.5-20.8 \mathrm{MeV}$, the area under the cross section curve of ${ }^{89} \mathrm{Y}(\mathrm{n}, 2 \mathrm{n}){ }^{88} \mathrm{Y}$ is smaller than in the second energy range of the same reaction. Moreover there is also an area under the 
curve of ${ }^{89} Y(n, 3 n){ }^{87} Y$ (figure 6). Since the measured amount of neutrons is proportional to the area under the cross section curves, the results in figure 1 and figure 3 are understandable.

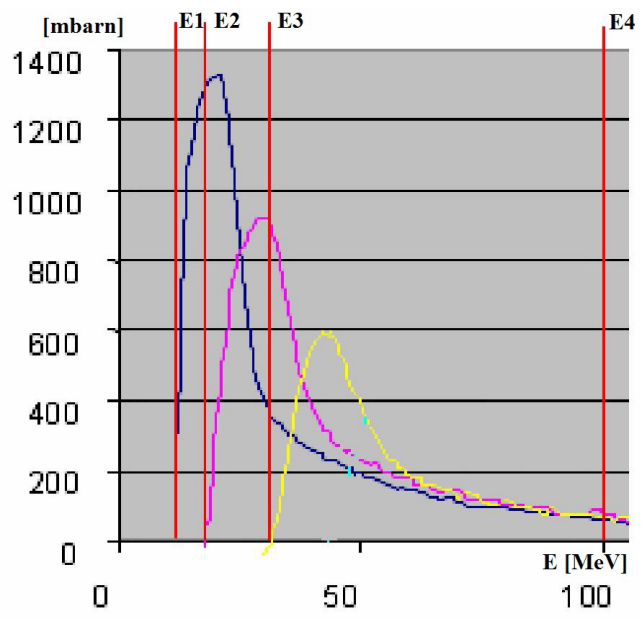

Figure 6. Cross sections of the three yttrium $(n, x n)$ reactions - threshold energies: $\mathrm{E} 1=11.5 \mathrm{MeV}{ }^{88} \mathrm{Y}$, E2 $=$ $20.8 \mathrm{MeV}^{87} \mathrm{Y}, \mathrm{E} 3=32.7 \mathrm{MeV}^{86} \mathrm{Y}$. TALYS calculation [6].

The above explanation for the experimental data presented in figure 4 and figure 5 is not sufficient. Calculations using the Monte Carlo method [1] show that in the energy range (20.8-32 MeV) the neutron fluence is higher than in the range $11.5-20.8 \mathrm{MeV}$ for the energy of the deuteron beam equal to $1 \mathrm{GeV}$ (figure 7).

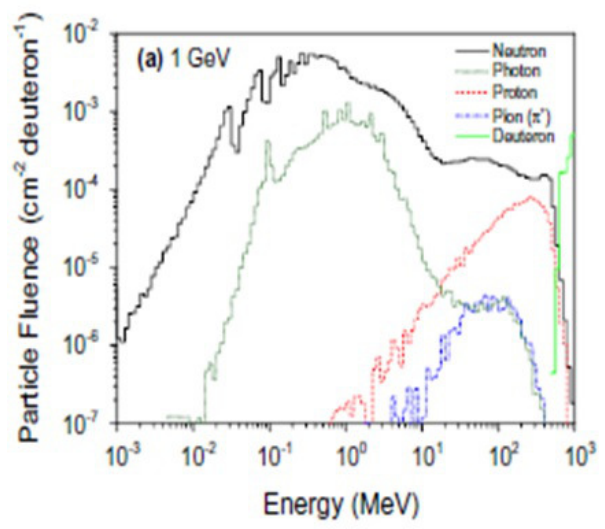

Figure 7. Neutron spectrum as a function of energy (black solid line) for the deuteron beam with an energy of 1 $\mathrm{GeV}$ [7].

Follow the same article [7]. Calculations using the Monte Carlo method show that in the three energy ranges (11.5-20.8 MeV, 20.8-32 MeV and 32-100 MeV) the neutron fluence is continuously decreasing with the neutron energy for the energy of the deuteron beam equal to $4 \mathrm{GeV}$ (figure 8). This explains the behavior of the neutron flux density presented in figure 4 and figure 5 . 


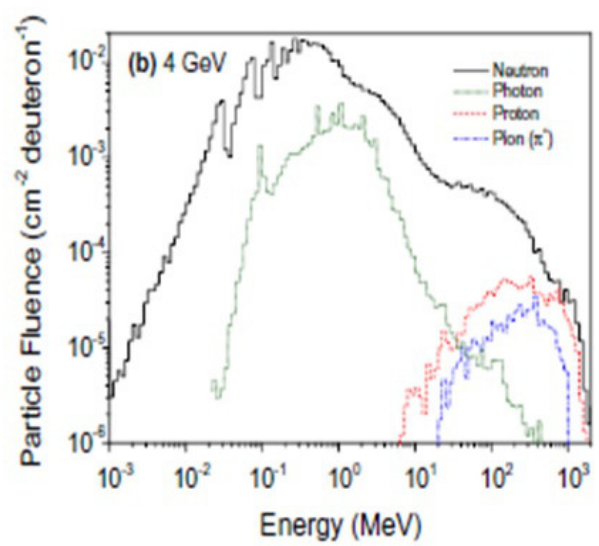

Figure 8. Neutron spectrum as a function of energy (black solid line) for the deuteron beam with an energy of 4 $\mathrm{GeV}[7]$.

\section{Conclusions}

This brief analysis carried out above let us to infer that the neutron flux density in the range above 10 $\mathrm{MeV}$ should be our concern in the future experimental research.

Theoretical analysis carried out by the authors [7] using MC method explains to some extent our experimental observation.

The tendencies presented above are vague and need to be checked in next experiments because we do not see clearly this effect in all our experimental results.

Though the measured amount of neutrons proportional to the area under the cross section curves in the energy range $11.5-20.8 \mathrm{MeV}$ explains the results for proton or deuteron beams in the range from 0.66 to $2 \mathrm{GeV}$, it does not explain the behavior of the average neutron flux for an energy of different beams higher than $2 \mathrm{GeV}$.

The measurement of the high neutron flux density and explanation of the results still need to be developed by an additional experiments and calculation.

Acknowledgments: We would like to thank the personnel of Dubna laboratories and "E+T RAW" collaboration.

\section{References}

[1] M. Bielewicz et al. Phys. Soc. 59(2), 2014 (2011)

[2] J. Frana Radioanal. and Nucl. Chem. 257, 583 (2003)

[3] M. Bielewicz et al. Nuclear Data Sheets 119, 296-298 (2014)

[4] E\&T RAW - Collaboration, Communication of the JINR, E1-2010-61 (2010)

[5] W. Furman et al., PoS (Baldin ISHEPP XXI) 086. www.pos.sissa.it (2013)

[6] A. J. Koning, S. Hilaire, M. C. Duijvestijn, TALYS Santa Fe, AIP Conference Proceedings 769, 1154-9 (2005)

[7] N. L. Asquith et al., Annals of Nuclear Energy 63, 742-750 (2014) 